\title{
Noncrossing Hamiltonian Paths in Geometric Graphs
}

\author{
Jakub Černý, Zdeněk Dvořák, Vít Jelínek, and Jan Kára \\ Department of Applied Mathematics \\ Charles University \\ Malostranské náměstí 25, 11800 Praha 1 \\ $\{$ kuba, rakdver, jelinek, kara\}@kam.mff .cuni.cz
}

\begin{abstract}
A geometric graph is a graph embedded in the plane in such a way that vertices correspond to points in general position and edges correspond to segments connecting the appropriate points. A noncrossing Hamiltonian path in a geometric graph is a Hamiltonian path which does not contain any intersecting pair of edges. In the paper, we study a problem asked by Micha Perles: Determine a function $h$, where $h(n)$ is the largest number $k$ such that when we remove arbitrary set of $k$ edges from a complete geometric graph on $n$ vertices, the resulting graph still has a noncrossing Hamiltonian path. We prove that $h(n)=\Omega(\sqrt{n})$. We also determine the function exactly in case when the removed edges form a star or a matching, and give asymptotically tight bounds in case they form a clique.
\end{abstract}

\section{Introduction}

A geometric graph is a graph drawn in the plane so that its vertices are represented by points in general position (i.e., there are no three collinear points) and its edges are straight-line segments connecting the corresponding vertices.

Lately geometric graphs have been intensively studied. There are many papers studying the smallest number of edges needed to guarantee the occurence of some fixed subconfiguration in any geometric graph (the trivial result of this type following from Euler's polyhedral formula is that any geometric graph with at least $3 n-5$ edges must have two edges which intersect). Interesting results of this sort may be found in [1], [4], [5], [6], [7] to name a few or in the surveys on geometric graphs ([2], [3]).

In our paper we study the existence of a noncrossing Hamiltonian path (i.e. Hamiltonian path which does not cross itself) in a given geometric graph. In particular we concentrate on a problem presented by Micha Perles on DIMACS Workshop on Geometric Graph Theory in 2002, which asks to determine the largest possible number $h(n)$ such that every geometric graph on $n$ vertices with at least $\left(\begin{array}{l}n \\ 2\end{array}\right)-h(n)$ edges has a noncrossing Hamiltonian path. Apart from improving the asymptotic bounds of $h(n)$, we also focus on the restriction of the problem to some classes of geometric graphs. Let $h_{1}(n)$ denote the largest $k$ such that when we remove the edges of an arbitrary complete subgraph of size at most $k$ from a complete geometric graph on $n$ vertices, the resulting graph always has a noncrossing Hamiltonian path. We prove that there exist constants $0<c_{1}<c_{2}$ such that $c_{1} \cdot \sqrt{n}<h_{1}(n)<c_{2} \cdot \sqrt{n}$ (Theorems1 and3). Let $h_{2}(n)$ denote the largest $k$ such that when we remove an arbitrary star with at most $k$ edges from a complete geometric graph on $n$ vertices, the resulting graph still has a noncrossing 
Hamiltonian path. In Theorem 6 we show that $h_{2}(n)=\lceil n / 2\rceil-1$. We also prove that $h_{3}(n)=\lceil n / 2\rceil-1$, where $h_{3}(n)$ is the largest $k$ such that when we remove at most $k$ arbitrary edges from the complete geometric graph on $n$ vertices in convex position, then the graph still has a noncrossing Hamiltonian path (Theorems 4 and 5). Further we prove that when we remove any matching from a complete geometric graph, the resulting graph has a noncrossing Hamiltonian path (Theorem 2).

The paper is organized as follows: In section 2 we introduce basic definitions and notation. In sections 3 and 4 we study complete geometric graphs with removed complete subgraph and we prove the asymptotically tight bounds on the size of complete subgraph removed. In section 5 we prove the tight bounds on the number of edges removed from convex geometric graph and in section 6 we prove the tight bounds on the size of a star removed from a complete geometric graph.

\section{Definitions and Notation}

In this section we introduce basic definitions and notation used throughout this paper. A geometric graph $G$ is an ordered pair $(V, E)$ where $V$ is a set of points in general position in the plane (called vertices of $G$ ) and $E$ is a set of straight-line segments connecting two vertices (called edges of $G$ ). A Hamiltonian path in a graph $G$ is a path contained in $G$ which visits all the vertices of $G$. A noncrossing Hamiltonian path in a geometric graph $G=(V, E)$ is a Hamiltonian path which does not intersect itself. A convex hull of a set of points $X \subset R^{2}, X=\left\{x_{1}, \ldots, x_{n}\right\}$ is a set of points $H=\left\{h \in R^{2}: \exists a_{1}, \ldots a_{n}\right.$ such that $\forall i \in\{1, \ldots, n\} a_{i} \in R, a_{i} \geq 0, \sum_{i=1}^{n} a_{i}=$ 1 and $\left.h=\sum_{i=1}^{n} a_{i} x_{i}\right\}$. We say that a point $p$ lies below a line $l$ (line $l$ must not be parallel to $y$-axis) if it lies in the half-plane defined by $l$ which contains $-\infty$ on the $y$ axis. Similarly we use the term above a line. A point $u$ lies to the left of $v$ if the $x$ coordinate of $u$ is less than or equal to the $x$-coordinate of $v$. Similarly we define that $u$ is to the right of $v$. Let $s$ be a segment in $R^{2}$ defined by two endpoints $u, v \in R^{2}$, where $u$ is to the left of $v$. We say that a point $p \in R^{2}$ lies below the segment $s$ if it lies below a line defined by $u$ and $v$, to the right of $u$ and to the left of $v$. Let $Z \subset R^{2}, Z=\left\{z_{1}, \ldots, z_{n}\right\}$ be a set of points in the plane. An $x$-monotone order of $Z$ is an ordering of $Z$ in which the $x$-coordinates of the points form a monotone sequence. Analogously we define a $y$-monotone order of $Z$. A point $z \in Z$ is called an extremal point of $Z$ if it belongs to the boundary of the convex hull of $Z$. A segment $u v$, with $u$ and $v$ in $Z$, is called an extremal segment of $Z$ if it is a subset of the boundary of the convex hull of $Z$.

\section{The Lower Bound for Complements of Cliques}

In the following two sections we consider a particular class $\mathcal{C}$ of geometric graphs the complements of complete subgraphs. A geometric graph $G=(V, E)$ is in $\mathcal{C}$ iff there exist $X, Y \subseteq V$ such that $V=X \cup Y, X \cap Y=\emptyset$ and $E$ is the set of all the possible edges with at least one enpoint in $Y$ (i.e. $G$ is obtained from a complete graph by removing the edges of a complete subgraph). We prove that there exists a constant $c_{1}>0$ such that any geometric graph $G \in \mathcal{C}$ with $|X| \leq c_{1} \cdot \sqrt{|V|}$ has a noncrossing Hamiltonian path. 
Lemma 1. Let $G=(V, E)$ be a geometric graph, $G \in \mathcal{C}$. If there exists a line $l$ such that all the vertices from $X$ are in one half-plane defined by $l$ and at least $|X|$ vertices from $Y$ are in the other half-plane, then there exists a noncrossing Hamiltonian path in $G$.

Proof. We may WLOG assume that the line $l$ is parallel to the $y$-axis and that all the vertices from the set $X$ are in the left half-plane. We iterate the procedure described below to find a noncrossing Hamiltonian path in $G$. In each step, the procedure adds a new vertex to the path that it has constructed in the previous steps, according to the following rules: first, we take the upper extremal segment of the vertices not yet added to the path (the whole set $V$ in the beginning) which crosses $l$ (see Figure 1 on the left). If the last vertex added to the path or the left endpoint of the extremal segment is from $Y$, then the left endpoint of the segment is added to the path (Figure 1 on the right), otherwise we add the right endpoint (Figure 2 on the left). If no vertices were added yet, we may add either of the endpoints. If $l$ does not cross the convex hull, then we simply add the remaining vertices to the path in an $x$-monotone order. See Figure 2 on the right for an example of the path produced by this algorithm.

It is clear that the algorithm finishes when it adds all the vertices to the path. It is also easy to see that there are no two consecutive vertices from $X$ on the constructed path and hence it is indeed a path in $G$. The only place in the algorithm, where two vertices from $X$ might be added consecutively to the path, is when the convex hull of the vertices not yet on the path no longer intersects $l$, all these remaining vertices lie to the left of $l$, and the algorithm is adding them in an $x$-monotone order. But at that time there is at most one vertex from $X$ not on the path: the other vertices from $X$ were added in the previous steps because there are at least $|X|$ vertices from $Y$ in the right half-plane, and each of them has been added to the path immediately after a vertex from $Y$, with the possible exception of the first vertex of the path.

It remains to prove that the path is noncrossing. We check that after each step of the algorithm, the path does not intersect the convex hull of the remaining vertices including the vertex just added to the path. From this it is obvious that the path does not intersect itself (at each vertex each of the following edges of the path must lie in the convex hull and the previous edges lie outside of it). When the path contains only one vertex, the claim is obviously true. When we add a new vertex to the path, the edge connecting the new vertex to the previous vertex on the path cannot intersect the convex hull of the remaining vertices - if the edge intersected the convex hull, then the previous vertex on the path would have to lie in the lower half-plane defined by the upper extremal segment of the remaining vertices intersecting $l$ (see Figure 3). But then we get contradiction with the choice of the vertex in the previous step of the algorithm (the vertex cannot be an endpoint of the upper extremal segment intersecting $l$ ). From the induction we know that no other edge of the path can intersect the convex hull of the remainig vertices and so we have proven that the path does not intersect itself.

Now we prove a similar result for a general choice of the sets $X$ and $Y$ :

Theorem 1. Let $G=(V, E)$ be a geometric graph, $G \in \mathcal{C}$. If $|Y| \geq 2 \cdot|X| \cdot(|X|+1)$ then there exists a noncrossing Hamiltonian path in $G$. 

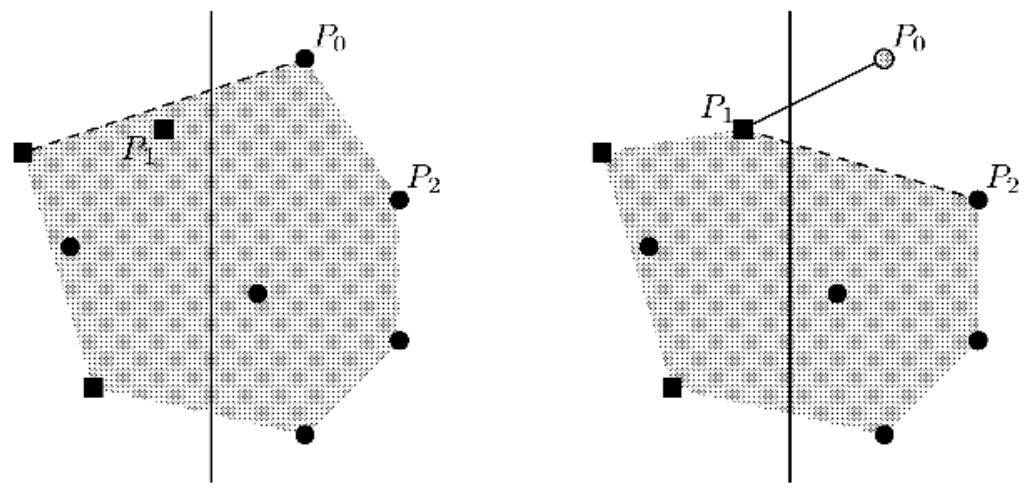

Fig. 1. The left figure shows the first step of the algorithm. The right figure shows the second step of the algorithm — add left end of the segment to the path.
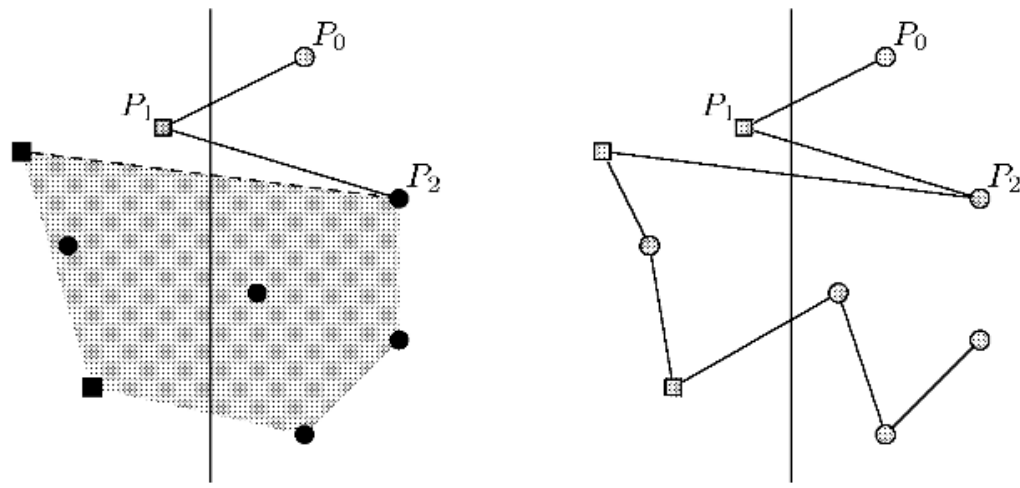

Fig. 2. On the left is the third step of the algorithm - add right end of the segment to the path. On the right is the whole noncrossing Hamiltonian path.

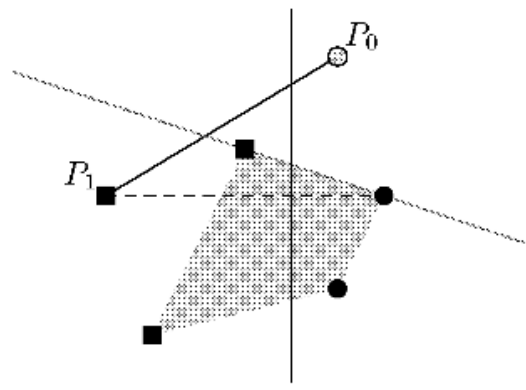

Fig. 3. If the segment to new vertex intersected the convex hull of the remaining vertices the previous vertex on the path would have to lie in the lower half-plane. 


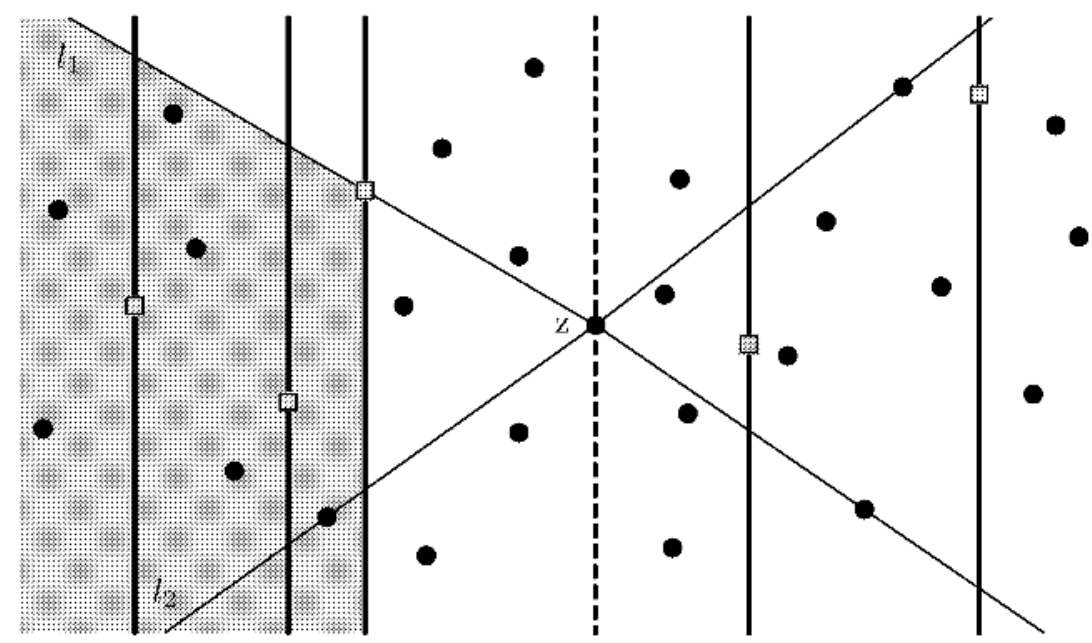

Fig. 4. Partitioning of the plane into the strips and choice of the vertices to which Lemma 1 should be applied.

Proof. We can WLOG assume that there are no two vertices from $V$ with the same $x$ coordinate. Now consider the partitioning of the plane into $|X|+1$ vertical strips separated by vertical lines passing through the points of $X$ (see Figure 4). From the pigeonhole principle it follows that there is a strip $S$ with at least $2 \cdot|X|$ vertices from $Y$ in it. Let $x_{l}$ denote the number of vertices from $X$ to the left of $S$ (including the vertex on the left boundary of $S$ ). Similarly let $x_{r}$ denote the number of vertices of $X$ to the right of $S$. Now we can certainly choose a vertex $z$ from $S$ such that there are at least $2 \cdot x_{l}$ vertices in $S$ to the left from $z$ and at least $2 \cdot x_{r}$ vertices in $S$ to the right from $z$. The vertical line passing through $z$ splits $S$ into two strips, denoted $S_{l}$ (the left one) and $S_{r}$ (the right one).

We now describe a procedure to find a noncrossing path starting in $z$ and containing all the vertices to the left of $z$. We find lines $l_{1}, l_{2}$ such that $z \in l_{1} \cap l_{2}$, both $l_{1}$ and $l_{2}$ contain some vertex lying to the left from $S_{l}$ and there is no vertex lying to the left from $S_{l}$ which would lie above $l_{1}$ or below $l_{2}$ (see Figure 4). It is clear that in $S_{l}$ there are either at least $x_{l}$ vertices below $l_{1}$ or at least $x_{l}$ vertices above $l_{2}$. Lets WLOG assume that the former is true. Let $Z$ denote the set of vertices lying to the left from $z$ and below $l_{1}$, including the two vertices on the line $l_{1}$. Now we can apply Lemma 1 to the set $Z$ (line $l$ from the statement of the lemma is the left boundary of the strip $S$ ). From the lemma we get a noncrossing path starting in $z$ containing all the vertices from $Z$ (note that $z$ is an endpoint of the upper extremal segment of $Z$ intersecting $l$ ). Because there are no vertices from $Z$ above the first segment of the path (all the vertices must lie below a line defined by the extremal segment from the second step of the procedure from Lemma 1 and the whole area above the first segment of the path lies above this line - see Figure 5), we can replace the first segment of the path by the path going through all the vertices in $S_{l}$ above the line $l_{1}$ in an $x$-monotone order (see Figure 6). By the 
replacing we got a noncrossing path starting in $z$ and using all the vertices of $V$ to the left of $z$. Similarly we can get a path starting from $z$ and containing all the vertices of $V$ to the right of $z$, then join these two paths in $z$ and get a noncrossing Hamiltonian path for $G$.

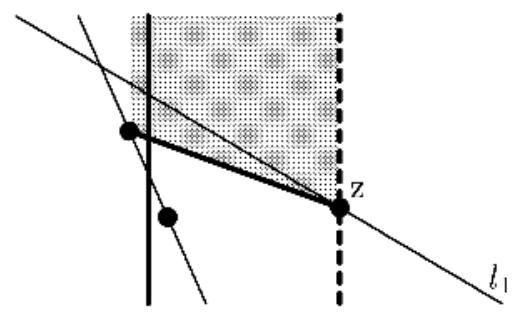

Fig. 5. There are no vertices lying above the first segment of the path and below the line $l_{1}$

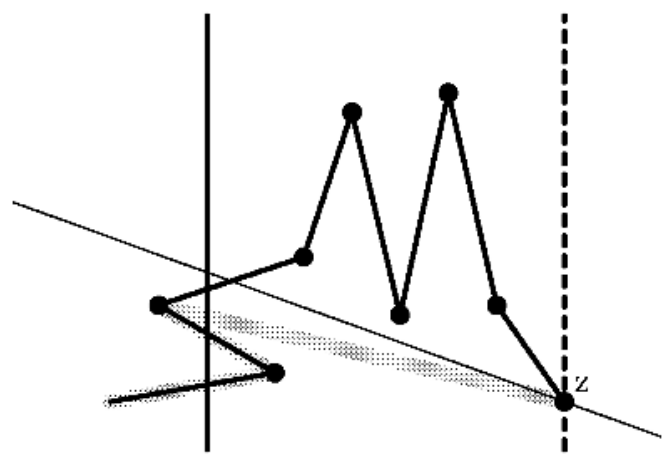

Fig. 6. Replacing the first segment of the path with an $x$-monotone path

Now using the Theorem 1 we can prove the following corollary:

Corollary 1. Let $G=(V, E)$ be a geometric graph and let $k=\left(\left(\begin{array}{c}|V| \\ 2\end{array}\right)-|E|\right)$ (i.e., $k$ is the number of edges not present in $G)$. If $2 \cdot 2 \cdot k \cdot(2 \cdot k+1) \leq|V|-2 \cdot k$ then there exists a noncrossing Hamiltonian path in $G$.

Proof. The idea of the proof is easy. We just use Theorem 1 for the original graph with removed edges of the complete subgraph containing all the edges missing in $G$.

More formally, let $X$ be the set of all vertices from $V$ with degree less than $|V|-1$. The size of $X$ is clearly less than or equal to $2 \cdot k$. Let $Y=V \backslash X$. From the statement of the corollary we know that $|Y| \geq 2 \cdot|X| \cdot(|X|+1)$ and hence the assumptions from 
the statement of Theorem 1 are satisfied and we can conclude that $G$ has a noncrossing Hamiltonian path. This gives a lower bound of order $\Omega(\sqrt{n})$ for the function $h(n)$ defined in the introduction.

Using the algorithm with a similar idea as the algorithm in Lemma 1 we can also prove the following result for the complements of matchings:

Theorem 2. Let $G=(V, E)$ be a geometric graph which is a complement of a matching (i.e. a graph with minimum degree $|V|-2$ ) and $|V| \geq 3$. Then $G$ has a noncrossing Hamiltonian path.

Proof. The case when $|V|=3$ is trivial so we can assume $|V| \geq 4$. We use the following algorithm for a construction of a noncrossing Hamiltonian path: first take any extremal point of $V$ to be the first vertex of the path. Let $x$ be the last vertex on the path constructed so far. Then at each step we choose a vertex $y$ which is an extremal point of the remaining vertices such that $\{x, y\}$ is an edge in $G$ which does not intersect the convex hull of the remaining points, and we add $y$ to the path. The vertex $y$ with the desired properties always exists if there are at least two remaining vertices. Let $H^{\prime}$ denote the set of points outside the path in the previous step of the algorithm and $H$ the set of points outside the path in the current step. In the previous step, $x$ was an extremal vertex of $H^{\prime}$ and so in the current situation there must be some segment $y_{1} y_{2}$ which is an extremal segment of $H$, such that neither of segments $x y_{1}$ and $x y_{2}$ do intersect the convex hull of $H$. Because at least one of these segments must be an edge in $G$ (its complement was a matching) we have just proven the existence of $y$.

If there is only one remaining vertex and it is not connected by an edge to the last point on the path, we cannot finish the path. Let $y_{1}$ be the last remaining vertex and $y_{2}, y_{3}$, and $y_{4}$ be the last vertices on the path in the reverse order (remember that $|V| \geq 4$ ). In this situation we remove the vertices $y_{2}$ and $y_{3}$ from the path to get the situation from figure 7 Because $y_{1}$ and $y_{2}$ are not connected by an edge of $G$, we know that $y_{4}$ must be connected by an edge to both $y_{1}$ and $y_{2}$ and one of these edges does not intersect the convex hull of the remaining points. So we can WLOG add $y_{1}$ to the path and then finish the path by adding $y_{3}$ and $y_{2}$.

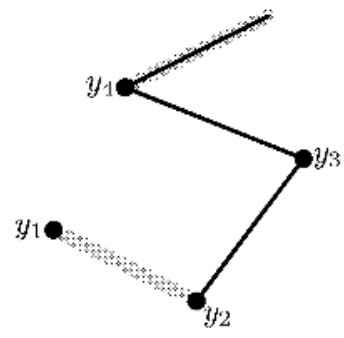

Fig. 7. The situation when we cannot add the last point to the noncrossing path. 


\section{The Upper Bound}

In this section we prove that there exist geometric graphs in $\mathcal{C}$ such that the size of $X$ is $O(\sqrt{|V|})$ and the graphs do not have a noncrossing Hamiltonian path. By proving this we get two asymptotically tight bounds on the function $h_{1}(n)$ defined in the introduction.

Lemma 2. Let $G=(V, E)$ be a geometric graph such that all the vertices of $V$ are in the convex position. Let $P=\left(v_{i_{1}}, \ldots, v_{i_{n}}\right)$ be a noncrossing Hamiltonian path in $G$. Then for any $j \in\{1, \ldots, n-2\}$ it holds that from the three vertices $v_{i_{j}}, v_{i_{j+1}}, v_{i_{j+2}}$ at least two are neighbors on the boundary of the convex hull of $V$ (i.e. at least two of them are connected by an extremal segment of $V$ ).

Proof. Assume that for some $j$ the statement does not hold. Then the vertices on the convex hull are split into three nonempty contiguous blocks of neighbouring vertices. But the noncrossing path can enter only two of these three parts (once the path enters some part it cannot leave it without crossing itself) and hence such a path cannot be Hamiltonian.

Theorem 3. For each $n_{0} \in N$ there exists $n \in N, n_{0} \leq n$ such that there is a geometric graph $G=(V, E), G \in \mathcal{C},|V|=n$ satisfying $|X|<3 \cdot \sqrt{n}$ without a noncrossing Hamiltonian path.

Proof. Lets have some $n_{0}$. Let $n$ be the smallest natural number greater than $n_{0}$ which is the square of some natural number. Now we describe a geometric graph on $n$ vertices with the desired properties. We place $n$ vertices of the graph on a circle with equal distances between neighbouring vertices. Then we split the vertices into $\sqrt{n}$ groups (each of size $\sqrt{n}$ ) in such a way that each group forms a contiguous sequence on the circle. Now we define the partitioning of $V$ into $X$ and $Y$ (and by this we determine the edges of the graph). We choose arbitrarily one group (let us call it $g$ ) and put all its vertices to $X$. In the other groups we put two vertices on the borders to $X$ and the remaining points to $Y$ (see Figure 8). Clearly $|X|=(\sqrt{n}-1) \cdot 2+\sqrt{n}<3 \cdot \sqrt{n}$, so it only remains to prove that the graph does not have a noncrossing Hamiltonian path.

Lets consider the first vertex $u$ in $g$. Because $u \in X$, it must be connected to some vertex $v$ from $Y$ by an edge of the path. We write $g^{\prime}$ for the group containing the vertex $v$. Because both neighbors of $u$ on the convex hull are also from $X, v$ cannot be a neighbor of $u$. From this we can also trivially conclude that neither $u$ nor $v$ can be the endpoints of the Hamiltonian path. Now we focus our attention on the part of the path which should contain all the remaining vertices of $g$. From Lemma 2 we know that the path must go from $v$ either to some other vertex of $g^{\prime}$ or to the neighbor of $u$ in $g$. From any vertex of $g$ the path must return back to $g^{\prime}$ to the neighbor of the vertex last used in $g$. From this we get that on the noncrossing Hamiltonian path there must be an alternation of vertices from $g$ and from $g^{\prime}$ in such a way that between any two vertices from $g$ there must be a vertex from $g^{\prime} \cap Y$. But we have $\sqrt{n}$ vertices from $g$ and only $\sqrt{n}-2$ vertices from $g^{\prime} \cap Y$ and so this is impossible. 


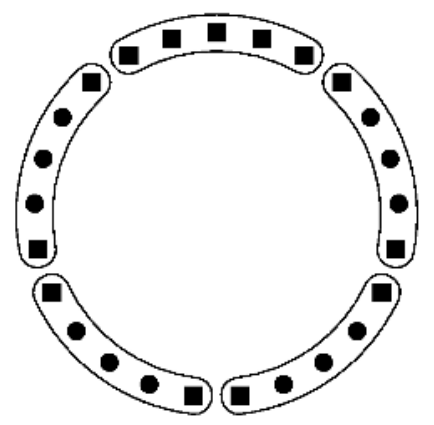

Fig. 8. Construction of a graph on $n$ vertices with $|X|=O(\sqrt{n})$ without a noncrossing Hamiltonian path

\section{Vertices in Convex Position}

In the following section we consider a class $\mathcal{D}$ of convex geometric graphs. A geometric graph $G=(V, E)$ is in $\mathcal{D}$ iff the vertices of $G$ are in a convex position. We show that if we remove $\lceil|V| / 2\rceil-1$ edges from the complete geometric graph then the noncrossing Hamiltonian path still exists (Theorem 4) but if we remove $\lceil|V| / 2\rceil$ edges it need not exist (Theorem 5). Note that the bounds are tight.

Theorem 4. Let $G=(V, E)$ be a geometric graph, $G \in \mathcal{D}, n=|V|$. Let $\bar{G}=(V, F)$ be the complement of $G$. If $|F| \leq\left\lceil\frac{n}{2}\right\rceil-1$ then there exists a noncrossing Hamiltonian path in $G$.

Proof. Let $v_{0}, v_{1}, \ldots, v_{n-1}$ be the vertices of $G$ in clockwise order, starting with an arbitrary one. Consider the complete geometric graph $G^{\prime}=(V, E \cup F)$. Let $P_{i}$ be the path $v_{i}, v_{i+1}, v_{i-1}, v_{i+2}, v_{i-2}, \ldots$ (counting the indices modulo $n$ ) in $G^{\prime}$. We observe that the paths $P_{1}, \ldots, P_{\lfloor n / 2\rfloor}$ are pairwise disjoint noncrossing Hamiltonian paths in $G^{\prime}$. Since $|F| \leq\left\lceil\frac{n}{2}\right\rceil-1$ we are done for $n$ even — at least one of the paths must avoid $F$ and hence it is a noncrossing Hamiltonian path in $G$. If $n$ is odd we observe that there are $\lfloor n / 2\rfloor$ edges $\left\{v_{0}, v_{n-1}\right\},\left\{v_{1}, v_{n-2}\right\}, \ldots,\left\{v_{\lfloor n / 2\rfloor-1}, v_{\lfloor n / 2\rfloor+1}\right\}$ which are not in any $P_{i}$. Let $A$ denote this set of edges. We can assume WLOG that the set $V$ forms the vertex set of a regular convex $n$-gon. Observe that every edge of $G^{\prime}$ can be mapped to an edge of $A$ by rotating $G^{\prime}$ along its centre of rotational symmetry. So we can WLOG assume that at least one of the edges of $F$ is in $A$ (and hence is not in any of the paths $\left.P_{i}\right)$. Now we can conclude using the same argument as for $n$ even that one of the $P_{i} \mathrm{~s}$ is a noncrossing Hamiltonian path in $G$.

Theorem 5. For each $n, n \geq 2$ there exists a geometric graph $G_{n}=\left(V_{n}, E_{n}\right)$ such that

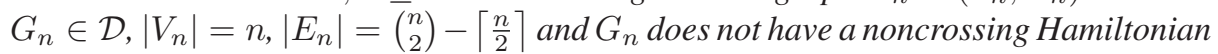
path.

Proof. Let $v_{0}, v_{1}, \ldots, v_{n-1}$ be the vertices of $G_{n}$ in clockwise order, starting with an arbitrary one. First we make an easy observation: the first (and the last) edge of a 
noncrossing Hamiltonian path $P$ is an extremal segment of $V$. Consequently, if $v_{i} v_{j}$ is an edge of such a path but not an extremal segment, then $P$ contains at least one extremal segment from each of the intervals $v_{i} \ldots v_{j}$ and $v_{j} \ldots v_{i}$.

Let $k=\left\lceil\frac{n}{2}\right\rceil$. We choose $F_{n}=\left\{\left\{v_{0}, v_{1}\right\},\left\{v_{1}, v_{2}\right\}, \ldots,\left\{v_{k-1}, v_{k}\right\}\right\}$ and $E_{n}$ as the complement of $F_{n}$. Let $B=\left\{v_{0}, \ldots, v_{k}\right\}$. Suppose there exists a noncrossing Hamiltonian path $P$ avoiding $F_{n}$. No edge in $P$ may join two points of $B$, as then by the observation above it would have to contain an edge from $F_{n}$. Therefore $B$ is an independent set of $P$, which is impossible as the largest independent set of $P$ is of size $k$.

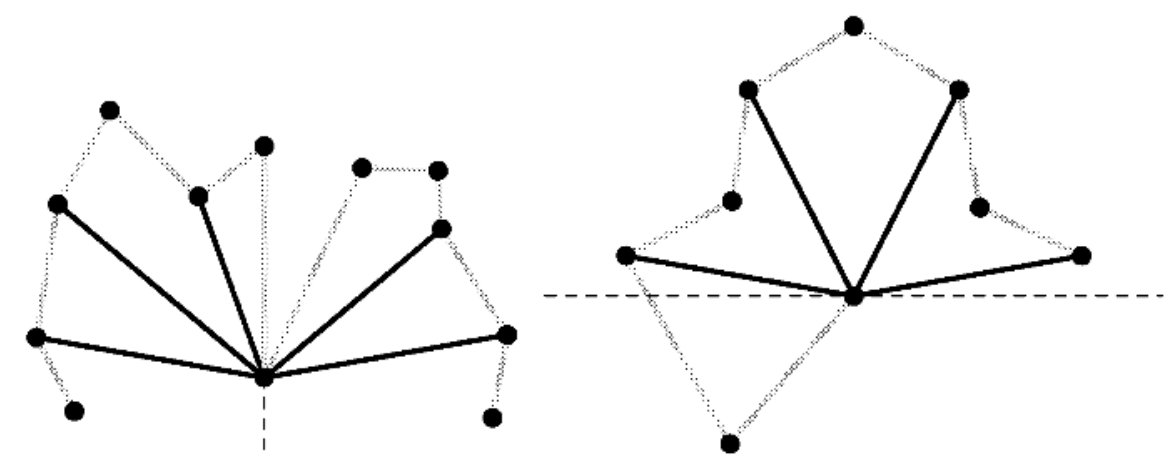

Fig. 9. Construction of a noncrossing Hamiltonian path. There are either two uncovered vertices in one of the cones (on the left) or every cone contains exactly one uncovered vertex (on the right).

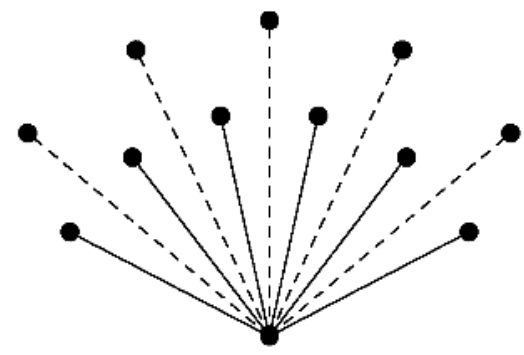

Fig. 10. Complement of a star without a noncrossing Hamiltonian path.

\section{Complement of Star}

In this section we consider a class of geometric graphs $\mathcal{S}$. A geometric graph $G=(V, E)$ is in $\mathcal{S}$ iff $E$ is a complement of $F$, where $F$ is the edge set of a star $K_{1, k}$. We prove 
that for $k \leq\lceil|V| / 2\rceil-1$ there always exists a noncrossing Hamiltonian path but for $k \geq\lceil|V| / 2\rceil$ it need not exist.

Theorem 6. For any geometric graph $G=(V, E)$ on $n$ vertices, $G \in \mathcal{S}$ such that $|F| \leq\left\lceil\frac{n}{2}\right\rceil-1$ there exists a noncrossing Hamiltonian path in $G$. For any $n, n \geq 2$ there exists a geometric graph on $n$ vertices $G_{n}, G_{n} \in \mathcal{S}$ with $\left|F_{n}\right|=\left\lceil\frac{n}{2}\right\rceil$ such that there is no noncrossing Hamiltonian path in $G_{n}$.

Proof. Let $C$ be the center of a star $F$, where $F$ has at most $\left\lceil\frac{n}{2}\right\rceil-1$ edges. We partition the plane into cones by extending the edges of $F$ into rays starting in $C$. If there is a cone that contains at least 2 vertices that are not covered by $F$, we use the construction from Figure 9 (left). Otherwise there must be exactly one vertex in each of the cones. At most one of the cones spans an angle greater than straight, let $x$ be the vertex inside this cone if such a cone exists, otherwise let $x$ be an arbitrary vertex not covered by $F$. The half-line starting in $C$ passing through $x$ splits its cone into two, so at least one of them spans an angle smaller than straight. Now we use the construction from Figure 9 (right).

We proceed to prove the second claim of the theorem. Let $V_{n}$ and $F_{n}$ look as in Figure 10. Note that the boundary of the convex hull of $V_{n}$ contains the vertex $C$, two edges of $F_{n}$, and all the vertices not covered by $F_{n}$. Moreover, every cone defined by the rays extending the edges of $F_{n}$ contains at most one vertex not covered by $F_{n}$.

Suppose there is a noncrossing Hamiltonian path $P$. If $C$ is the first vertex of $P$, then the second vertex of $P$ is one of the uncovered ones, the third one belongs to one of the half-planes determined by the first edge and $P$ cannot get to the other half-plane without intersecting its first edge, so the path cannot be Hamiltonian. Similarly, if $C$ is an interior vertex of $P$, the edges of $P$ adjacent to it split the remaining vertices into three nonempty parts, and $P$ cannot cover more than two of them without intersecting itself.

\section{Conclusion}

In this paper we proved that for several classes of geometric graphs (graphs with vertices in convex position, complements of stars, complements of cliques and complements of matchings) we need to remove $\Omega(n)$ edges from a complete geometric graph to forbid every noncrossing Hamiltonian path. This lets us conjecture that $h(n)=\theta(n)$ where $h(n)$ is the largest $k$ such that when we remove arbitrary set of $k$ edges from a complete geometric graph on $n$ vertices, the resulting graph still has a noncrossing Hamiltonian path.

Acknowledgement. The authors would like to thank Jan Kratochvíl and Pavel Valtr who brought the problem to our attention, discussed it with us and helped us with the preparation of this paper.

\section{References}

1. P. K. Agarwal, B. Aronov, J. Pach, R. Pollack, and M. Sharir: Quasi-planar graphs have a linear number of edges Combinatorica 17 (1997) 1-9 
2. J. Pach: Geometric graph theory, Surveys in combinatorics, 1999 (Canterbury), 167-200, London Math. Soc. Lecture Note Ser., 267, Cambridge Univ. Press, Cambridge, 1999.

3. J. Pach and P. K. Agarwal: Combinatorial Geometry, Wiley Interscience, New York, 1995

4. J. Pach, R. Radoičić, G. Tardos, and G. Tóth: Geometric graphs with no self-intersecting path of length three, Graph Drawing, Lecture Notes in Computer Science 2528, Springer-Verlag, Berlin, 2002, 295-311

5. R. Pinchasi and R. Radoičić: On the number of edges in geometric graphs with no selfintersecting cycle of length 4, Proc. 19th Annual Symposium on Computational Geometry, submitted

6. G. Tardos: On the number of edges in a geometric graph with no short self-intersecting paths, in preparation

7. P. Valtr: Graph drawings with no $k$ pairwise crossing edges, Graph Drawing (Rome), Lecture Notes in Computer Science, vol. 1353 (1997), 205-218 